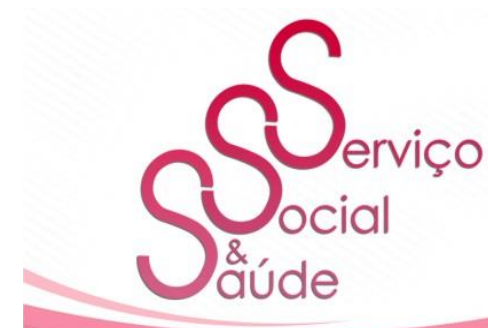

ISSN 2446-5992

(c) 10 (2)

10.20396/sss.v19i0.8665386

\title{
Gestão de alta hospitalar em Cuidados Continuados Integrados (CCI)
}

\author{
Hospital discharge mangement Integrated Continuous Care (CCI)
}

\author{
Priscilla Fernandes Fagundes ${ }^{1}$ \\ Estela Márcia Rondina Scandola ${ }^{2}$ \\ Keila Regina de Oliveira ${ }^{3}$
}

\section{RESUMO}

O tema deste estudo refere à gestão de alta hospitalar em Cuidados Continuados Integrados (CCI). O programa CCI tem o objetivo de atender pessoas que apresentem dependência funcional potencialmente susceptível de recuperação, por meio de uma abordagem integral que contemple as necessidades físicas, psíquicas, emocionais e sociais. A escolha pelo tema surgiu no período da Residência Multiprofissional em Saúde no Hospital Regional de Mato Grosso do Sul. O objetivo do trabalho é analisar o processo de gestão de alta hospitalar para a unidade CCI do Hospital São Julião a partir das entrevistas com os profissionais que compõem as Equipes de Gestão de Alta (EGAs) dos três (3) hospitais de alta complexidade que encaminham usuários para a unidade CCI. A metodologia utilizada foi do tipo qualitativo, a partir de entrevistas individuais com questões objetivas e outras abertas. Os sujeitos foram os profissionais de: Serviço Social, Medicina e Enfermagem que compõem as EGAs. Os dados foram organizados e analisados por meio da técnica de análise de conteúdo. Foram evidenciados três (3) eixos temáticos de discussão, sendo verificado que o fluxo de sinalização dos usuários é equivalente entre as EGAs e que os critérios utilizados para encaminhamento para o CCI podem ser conflituosos com os princípios do SUS. Nesse sentido, os trabalhadores em saúde possuem papel fundamental visando trazer em cena a defesa do SUS.

PALAVRAS-CHAVE: Gestão Hospitalar. Alta Hospitalar. Cuidados Continuados. Equipe

\footnotetext{
${ }^{1}$ Assistente Social. Pós-graduada em Cuidados Continuados Integrados/UFMS e Intensivismo/UNIDERP. Telefone: 5567 992791223. E-mail: priscilla_assistentesocial@ hotmail.com

${ }^{2}$ Assistente Social. Doutora em Serviço Social. Mestre em Saúde Coletiva. Escola Saúde Pública do Mato Grosso do Sul. E-mail: estelascandola@yahoo.com.br

${ }^{3}$ Assistente Social no Hospital Regional de Mato Grosso do Sul, Coordenadoria de Gestão do Trabalho. E-mail: koliveira@funsau.ms.gov.br
} 


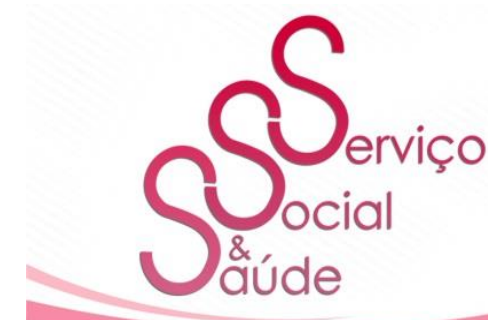

ISSN 2446-5992

(c) $10(0)$

10.20396/sss.v19i0.8665386

Interdisciplinar de Saúde.

\begin{abstract}
The theme of this study refers to hospital discharge management in Integrated Continuous Care (ICC). The CCI program aims to assist people with functional dependence potentially susceptible to recovery, through a comprehensive approach that addresses physical, psychological, emotional and social needs. The choice for the theme arose during the period of Multiprofessional Residency in Health at the Regional Hospital of Mato Grosso do Sul. The objective of the work is to analyze the discharge management process for the CCI unit of Hospital São Julião from the interviews with the professionals who make up the Discharge Management Teams (EGAs) of the three (3) highly complex hospitals that refer patients to the ICC. The methodology used was of a qualitative type, based on individual interviews with objective and open questions. The subjects were the professionals from: Social Work, Medicine and Nursing who make up the EGAs. The data were organized and analyzed using the content analysis technique. Three (3) thematic axes of discussion were evidenced, and it was verified that the flow of signaling from users is equivalent between the EMCs and that the criteria used for referral to the ICC can be in conflict with the principles of SUS. In this sense, health workers have a fundamental role in bringing about the defense of SUS.
\end{abstract}

KEYWORDS: Hospital Management. Hospital discharge. Continuous care. Interdisciplinary Health Team.

\title{
Introdução
}

$\boldsymbol{A}$ saúde enquanto política pública de seguridade social é assegurada na Constituição Federal como "direito de todos e dever do Estado" (BRASIL, 1988) e regulamentada como Sistema Único de Saúde (SUS), com vistas a "garantir às pessoas e à coletividade condições de bem-estar físico, mental e social" assegurando universalidade e integralidade (BRASIL, 1990). Diante do processo instituinte do SUS e no seu compromisso com a saúde dos idosos, o Hospital São Julião (HSJ) manifestou para a Secretaria Estadual de Saúde de Mato Grosso do Sul (SES/MS) interesse em exercer o papel de retaguarda aos hospitais de Alta Complexidade, a fim de liberar leitos de Urgência e Emergência (ALVARENGA, 2014).

Em 2011, o Ministério da Saúde instituiu os leitos de retaguarda e Unidades de Cuidados Prolongados (UCP) na Rede de Atenção às Urgências e Emergências no âmbito do SUS (BRASIL, 2011), sendo regulamentado no ano seguinte como "uma estratégia de cuidado intermediária entre os cuidados hospitalares de caráter agudo e crônico reagudizado e 


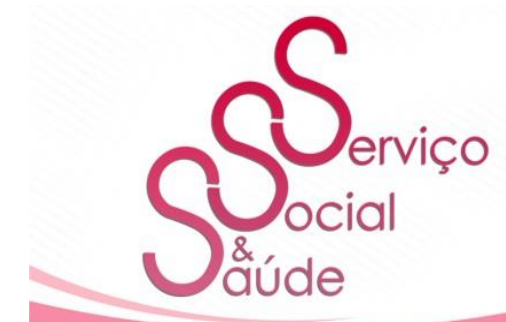

ISSN 2446-5992

(๑⿻)

10.20396/sss.v19i0.8665386

3

a Atenção Básica, inclusive a Atenção Domiciliar, prévia ao retorno do usuário ao domicílio" (BRASIL, 2012).

Nesse contexto em outubro de 2013 o HSJ foi escolhido pela SES/MS para sediar o Programa de Cuidados Continuado Integrados (CCI), pois já dispunha de leitos de retaguarda para os hospitais de alta complexidade. Além disso, conta com uma infraestrutura favorável para o trabalho multidisciplinar, com qualificação dos recursos humanos voltado à humanização no atendimento, características essas, primordiais para o modelo de atendimento em CCI, posto que a Política de Humanização é uma das bases do HSJ.

O modelo de atendimento CCI já desenvolvido em outros países como Portugal e Espanha, e em alguns estados brasileiros, tem o objetivo de atender pessoas que apresentam dependência funcional potencialmente susceptível de recuperação, por meio de uma abordagem integral que contemple as necessidades físicas, psíquicas, emocionais e sociais (DOLZ, 2013a). O Programa surgiu para atender as necessidades emergentes de saúde, dentre elas o envelhecimento da população, em virtude do aumento na expectativa de vida. Considerando as crescentes demandas dessa população foi instituída a Política Nacional de Saúde do Idoso que tem como meta promover uma "atenção à saúde adequada e digna para os idosos" (BRASIL, 2006).

Atualmente o programa CCI no estado de Mato Grosso do Sul é executado na Unidade Don Aldo Rabino do HSJ, com uma estrutura para atendimento de 22 leitos, financiados como leitos de UCP. Na unidade, os usuários contam com atendimento multiprofissional de: assistentes sociais, psicólogos, nutricionistas, enfermeiros, farmacêuticos, fisioterapeutas, médicos, técnicos e outros profissionais de apoio matricial, que atuam em conjunto com a pessoa internada e a família à reabilitação biopsicossocial, promovendo o máximo de independência aos usuários, e treinamento dos cuidadores/familiares.

$\mathrm{Na}$ metodologia de atendimento do programa CCI, o planejamento de alta dos hospitais de alta complexidade para a unidade CCI se dá por meio das denominadas "Equipes de Gestão de Alta (EGA)”, em consonância com a intitulação dada nos países europeus, as 


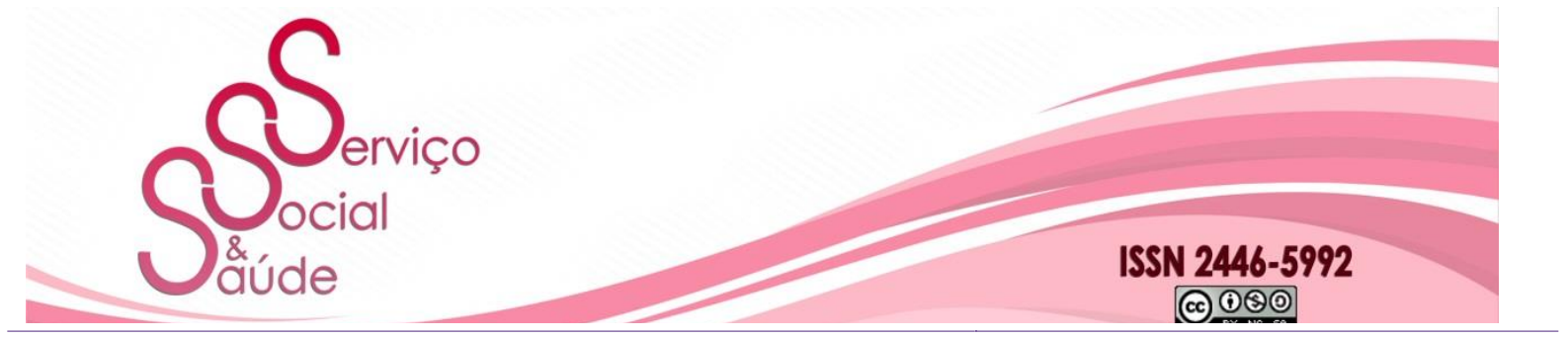

10.20396/sss.v19i0.8665386

4 quais são compostas por, no mínimo: um médico, um enfermeiro e um assistente social que são responsáveis pela preparação e gestão das altas hospitalares (PORTUGAL, 2006).

Compete as EGAs: facilitar a identificação e avaliação biopsicossocial precoce dos usuários que potencialmente possam requerer continuidade dos cuidados pós-alta hospitalar e recomendar opções para continuidade do cuidado, com base nas necessidades do paciente; colaborar no planejamento e facilitação de alta; estabelecer mediação com a rede de serviços a fim de promover o acesso aos recursos necessários, dentre outros (DOLZ, 2013a).

No contexto brasileiro, as competências da EGA são compatíveis ao Núcleo Interno de Regulação (NIR), que segundo a Política Nacional de Atenção Hospitalar (PNHOSP) é responsável pela "interface com as Centrais de Regulação [...] além de buscar vagas de internação, apoio diagnóstico e terapêutico fora do hospital para os pacientes internados" (BRASIL, 2013).

O Ministério da Saúde divulgou o "Manual de Implantação e Implementação do NIR", onde são pontuadas as principais atribuições do NIR, dentre elas: regular e gerenciar as diferentes ofertas hospitalares existentes; otimizar a utilização dos leitos hospitalares; aprimorar e apoiar o processo integral do cuidado ao usuário dos serviços hospitalares visando ao atendimento mais adequado às suas necessidades [...], sendo recomendado que a equipe mínima seja composta por médico, enfermeira e assistente social (BRASIL, 2017), assim como, as atribuições e equipe mínima da EGA.

Para a implantação do Programa foi realizada uma capacitação pela equipe do Hospital Samaritano em parceria com o Centro de Estudos Augusto Leopoldo Ayrosa Galvão (CEALAG) aos profissionais do HSJ e aos profissionais que fariam parte das EGAs dos hospitais de alta complexidade que sinalizam usuários para o programa, sendo eles: Hospital Regional de Mato Grosso do Sul (HRMS), Hospital Universitário Maria Aparecida Pedrossian (HUMAP) e da Associação Beneficente de Campo Grande - Santa Casa (ABCG).

Cada hospital possui uma EGA que identifica os usuários com critérios para o Programa, realiza as avaliações, e nos casos de pessoas com os critérios para a reabilitação, é 


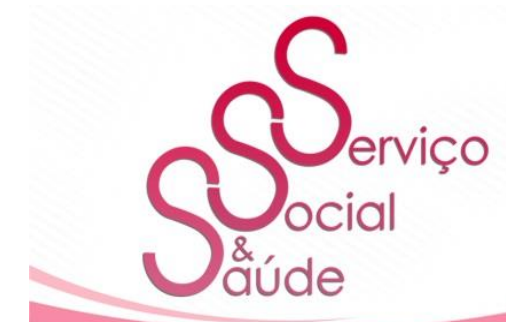

ISSN 2446-5992

(๑⿻)

10.20396/sSS.v19i0.8665386

5

encaminhado um relatório multiprofissional intitulado de "pré-alta". O HSJ executor do programa CCI tem uma equipe também denominada EGA que, com base nas informações da pré-alta dialoga com a EGA de origem visando disponibilizar ou não o leito para reabilitação.

Vale considerar que durante a capacitação do Programa, as equipes foram orientadas quanto ao fluxograma de encaminhamento para a unidade CCI, assim como, sobre os critérios de admissão no programa. Dessa forma, o presente estudo visa analisar o processo de gestão de alta nos diferentes hospitais de alta complexidade, após seis (6) anos da inauguração do programa CCI no estado.

\section{PROCEDIMENTOS METODOLÓGICOS}

Nesta pesquisa foi utilizado o estudo do tipo qualitativo que segundo Minayo (2009) considera o universo dos significados, crenças, valores que não podem ser quantificados, ou seja, ele parte da realidade social. Tal escolha metodológica se deu devido à proposta do estudo que é voltada a análise do processo de gestão de alta hospitalar em CCI a partir do discurso de diferentes profissionais em saúde.

A pesquisa foi realizada em três (3) hospitais de alta complexidade que atendem o SUS no município de Campo Grande, e que encaminham os usuários para o programa CCI do Hospital São Julião (HSJ), sendo eles: Hospital Regional de Mato Grosso do Sul (HRMS), Hospital Universitário Maria Aparecida Pedrossian (HUMAP) e Associação Beneficente de Campo Grande (ABCG - Santa Casa).

Os sujeitos envolvidos na pesquisa foram os profissionais de Serviço Social, Enfermagem e Medicina que compõem a EGA de cada hospital. Foram excluídos do estudo os profissionais da EGA do hospital São Julião (executor do programa CCI). Foi selecionado aleatoriamente, apenas um (1) profissional de cada área de cada hospital, totalizando nove (9) participantes.

A coleta de dados se deu a partir de entrevistas individuais com questões objetivas e outras abertas, permitindo o discurso do participante.

\begin{tabular}{|l|l|l|l|l|l|l|} 
Serv. Soc. \& Saúde & Campinas, SP & v. 19 & $1-25$ & e020010 & 2020 & e-ISSN 2446-5992 \\
\hline
\end{tabular}




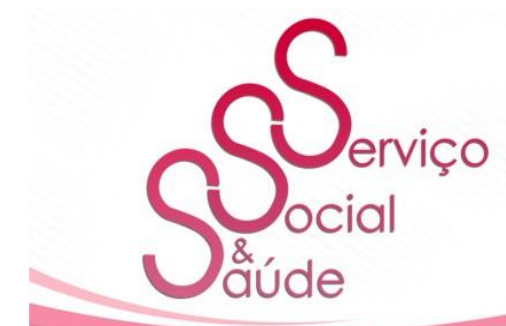

ISSN 2446-5992

(๑⿻)

10.20396/sss.v19i0.8665386

6

Foi realizado contato com os três (3) hospitais: HUMAP, HRMS e ABCG, a fim de verificar o interesse dos profissionais que compõem a EGA em participar da pesquisa, sendo programadas as entrevistas na data e horário de preferência de cada profissional.

As entrevistas foram realizadas separadamente, por meio de gravação de áudio, e posteriormente foram transcritas transformando-se em documento denominado "base de dados". Tiveram acesso ao conteúdo das entrevistas apenas a pesquisadora, orientadora e coorientadora.

Os dados coletados nas entrevistas foram transcritos e o conteúdo organizado por hospital. Nessa organização percebeu-se que havia a possibilidade de identificação dos hospitais, e respectivos participantes. Por isso, optou-se por identificá-los da seguinte forma: letras (A) assistente social, (M) médico e (E) enfermeiro, e pelos números 1, 2 e 3 aleatoriamente.

A partir de leituras sucessivas foram constatados conteúdos recorrentes nos discursos, que não estavam necessariamente nas mesmas questões. Por isso, foram analisados os conteúdos em todo o material, pois as respostas estavam em diferentes trechos da entrevista. Posteriormente foi realizada a exploração do material, onde foram definidos os eixos temáticos com base nos conteúdos similares das mensagens. Por fim, foi realizado o tratamento dos resultados, onde foram feitas as inferências e interpretação dos dados, ou seja, o momento em que o pesquisador realiza a análise reflexiva e crítica do conteúdo. Para isso, foi utilizada a técnica de análise de conteúdo que segundo Minayo (2009, p. 74), se refere à "verificação de hipóteses e/ou questões e descoberta do que está por trás dos conteúdos manifestos".

Destaca-se que a terminologia adotada para a pesquisa foi "usuário", visto que a expressão "paciente" remete a uma vertente biomédica, contrária a proposta deste estudo. No entanto, nas transcrições dos discursos dos participantes e nas citações manteve o termo original. 


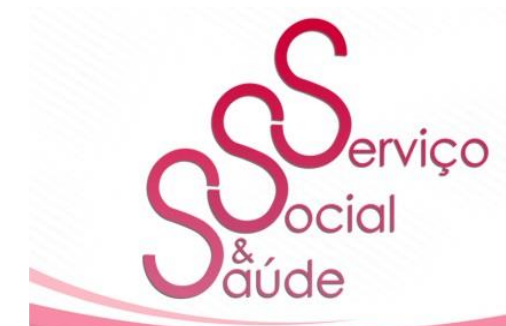

ISSN 2446-5992

(๑⿻)

10.20396/sSS.v19i0.8665386

Foi solicitada autorização da direção dos três hospitais HRMS, HUMAP e ABCG, para realização das entrevistas, após aprovação do projeto de pesquisa pelo Comitê de Ética em Pesquisa com seres humanos da Universidade Anhanguera-UNIDERP, cujo $\mathrm{n}^{\circ}$ de parecer foi: 3.318.507. Para a realização das entrevistas os participantes da pesquisa assinaram um termo de consentimento livre e esclarecido (TCLE), conforme regulamentado na resolução 466 de 12 de dezembro de 2012 (BRASIL, 2012).

\section{RESULTADOS E DISCUSSÕES}

Foram realizadas sete (7) entrevistas, com três (3) médicos, três (3) assistentes sociais e um (1) enfermeiro, pois dois (2) hospitais não dispunham de enfermeiro (a) na equipe de Gestão de Alta no período de coleta de dados. Em relação ao tempo em que fazem parte da EGA, quatro (4) profissionais participam da equipe desde sua implantação no ano de 2013 e três (3) informaram que estão na equipe em torno de dois (2) anos.

Com a base de dados constituída pela transcrição e trato das falas para se tornarem compreensíveis foram retirados elou inseridas partículas como "e", "né”, “ahn", "como", “então" [...], sendo que as leituras sucessíveis trouxeram em evidência eixos temáticos que possibilitaram recolher os discursos com conteúdo próximos. A partir desse trabalho apresentaram-se os resultados que seguem em três blocos de discussão:

\section{CRITÉRIOS USADOS PARA SINALIZAÇÃO DE USUÁRIOS PARA A UNIDADE CCI}

Ao serem questionados sobre os critérios utilizados por área profissional para sinalização de usuários para o Programa CCI, foram obtidos os seguintes discursos:

Essencialmente são pacientes com doenças agudas ou crônicas agudizadas e com possibilidade de reabilitação, com algum critério que permita a reabilitação do paciente (M1). 


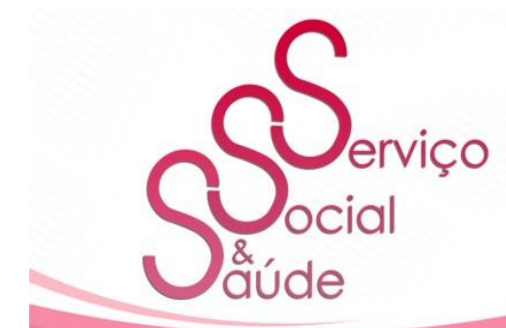

ISSN 2446-5992

(๑⿻)

10.20396/sss.v19i0.8665386

O paciente com perda aguda da autonomia, aquele paciente que mesmo que tenha uma doença crônica, mas interna por agudização dela [tenho] pela cabeça os critérios, mas o mais importante é aquele que além de ter uma perda aguda da autonomia, ele tenha um potencial grande de reabilitação (M2).

Geralmente eram pacientes que tinham a autonomia, para fazer as atividades do cotidiano, e, por conta do problema de saúde, eles ficaram com dependência. Dependência motora, dependência emocional, dependência nos cuidados mesmo (A1).

De acordo com os entrevistados M1, M3 e A1 um dos critérios para sinalização de usuários para o programa CCI é a perda da autonomia, termo esse derivado do grego auto, que significa próprio, e nomos, definido como lei, regra ou norma, sendo que a união desses dois termos resulta na ideia de autogoverno, autodeterminação, capacidade do ser humano decidir sobre si (ARAUJO; BRITO; NOVAES, 2008).

$\mathrm{Na}$ perspectiva da saúde, a autonomia inclui a liberdade de escolha, de ação e autocontrole sobre a vida, que depende não apenas do sujeito, mas de condições e fatores externos. Entende-se autonomia como a capacidade de lidar com a rede de dependências (CAMPOS; CAMPO, 2006).

Conforme o projeto de implantação dos cuidados continuados integrados, o perfil para atendimento no programa é "pacientes com incapacidade desencadeada por um processo agudo recente, com capacidade para colaborar em um programa de reabilitação e que é previsível uma melhora na capacidade funcional" (DOLZ, 2013b).

A ideia de autonomia citada pelos entrevistados "eram pacientes que tinham a autonomia, para fazer as atividades do cotidiano" (A1) e "paciente com perda aguda da autonomia" (M2), se mistura a ideia de independência funcional.

De acordo com Ribeiro e Scandola (2017) “ao definir a independência funcional como autonomia reduz-se essa a aspectos da vida diária sem considerar a capacidade de decidir e refletir sobre diferentes dimensões do viver como a participação efetiva das decisões sobre

\begin{tabular}{l|l|l|l|l|l|l|} 
Serv. Soc. \& Saúde & Campinas, SP & v. 19 & $1-25$ & e020010 & 2020 & e-ISSN 2446-5992 \\
\hline
\end{tabular}




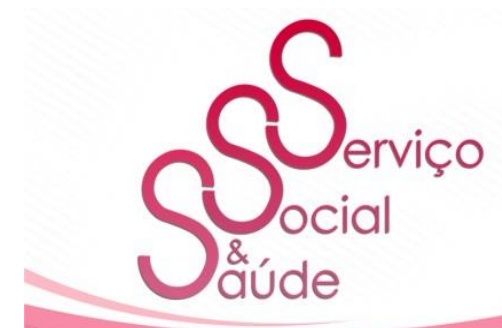

ISSN 2446-5992

(c) 10 (2)

10.20396/sSS.v19i0.8665386

9

sua vida". Portanto, compreende-se que para o usuário ter autonomia, ele não necessariamente precisa ser capaz de realizar as atividades do cotidiano de forma independente.

O entrevistado A1 especifica alguns tipos de dependências, decorrentes do processo de adoecimento, que são critérios para reabilitação na Unidade CCI, sendo elas: motora, emocional e nos cuidados. Essas condições estão de acordo com o proposto no Programa, que segundo Alvarenga (2014, p. 158) a reabilitação em "cuidados continuados integrados incorpora uma abordagem integral das necessidades e visão biopsicossocial".

No que se refere à ideia de pessoas com doenças agudas ou com doenças crônicas agudizadas, Mendes (2011) apresenta que "em geral, as condições agudas iniciam-se repentinamente; apresentam uma causa simples e facilmente diagnosticada; são de curta duração; e respondem bem a tratamentos específicos", e dentre as formas de tratamento, têmse a reabilitação, enfoque do programa CCI.

É perceptível que há distintas visões sobre o significado de reabilitação no conjunto das instituições que participam do CCI, pois o M1 citou que para sinalizar o usuário para o Programa CCI é necessário que conte "com algum critério que permita a reabilitação" e o M2 que "tenha um potencial grande de reabilitação".

De acordo com o protocolo instituído pelo Hospital São Julião, o objetivo geral do Programa é "proporcionar a gestão de paciente com estado funcional alterado, realizando reabilitação por meio de intervenções clínicas, psicossociais e funcionais através de equipe multiprofissional" (HSJ, 2017), objetivo esse que confere com o critério usado pelas EGAs entrevistadas. Ainda quanto aos critérios para sinalização, as assistentes sociais apresentaram outros conteúdos discursivos:

O Serviço Social avalia principalmente essa questão sociofamiliar, se esse paciente tem familiar que acompanha a internação. Nós analisamos principalmente, os vínculos familiares [...] porque esse processo de reabilitação tem a coparticipação da família. Essa família também precisa ser treinada, para poder cuidar desse paciente dentro da realidade social a qual se encontra (A1). 


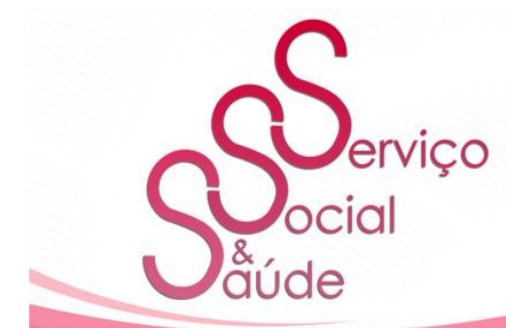

ISSN 2446-5992

(๑⿻)

10.20396/sss.v19i0.8665386

10

$\mathrm{Na}$ área do Serviço Social, um dos critérios primordiais é que o paciente tenha família. A família é muito importante, porque além deles já estarem aqui acompanhando o paciente, ele precisa estar como acompanhante lá no CCI para também ser o cuidador e ser treinado para quando vai para domicílio (A2).

Nós do Serviço Social, a gente utiliza a escala de GIJON, para estar fazendo essa classificação. A gente tenta adaptar essa escala para a nossa realidade, que a gente até tem uma dificuldade (A3).

Observa-se que o critério referente à rede de apoio sócio familiar foi relatado pelas assistentes sociais entrevistadas, como sendo um dos principais critérios da profissão para encaminhamento ao Programa, tal como o modelo europeu, o Brasil incorporou a corresponsabilização da família no processo de reabilitação, tendo o "doente e família como unidade de cuidado", promovendo o envolvimento dos cuidadores no processo de reabilitação (CARRARA; DOLZ, 2013).

Também a Política Nacional de Humanização (PNH) orienta que "incluir usuários e suas redes sócio familiares nos processos de cuidado é um poderoso recurso para a ampliação da corresponsabilização no cuidado de si” (BRASIL, 2013). A relação do profissional assistente social com a participação da família no processo de internação está embasada nos "Parâmetros para Atuação do Assistente Social na Política de Saúde”, em que uma das ações a serem desenvolvidas pelo assistente social no âmbito da saúde é "fortalecer os vínculos familiares, na perspectiva de incentivar o usuário e sua família a se tornarem sujeitos do processo de promoção, proteção, prevenção, recuperação e reabilitação da saúde" (CFESS, 2010, p. 45). Esse processo de “emancipação” está em estreita relação com a autonomia.

No que concerne ao relato do entrevistado A3 sobre a escala de Gijon, esse instrumento objetiva "avaliar a condição sociofamiliar dos idosos, por meio dos seguintes domínios: situação familiar, situação econômica, habitação, relações sociais e apoio da rede social” (DOMINGUES, et al., 2012, p. 344), embora não seja validado no Brasil, esse instrumental é utilizado no contexto do CCI brasileiro, conforme adotado na RNCCI de

\begin{tabular}{r|r|r|r|r|r|r}
\hline Serv. Soc. \& Saúde & Campinas, SP & v. 19 & $1-25$ & e020010 & 2020 & e-ISSN 2446-5992 \\
\hline
\end{tabular}




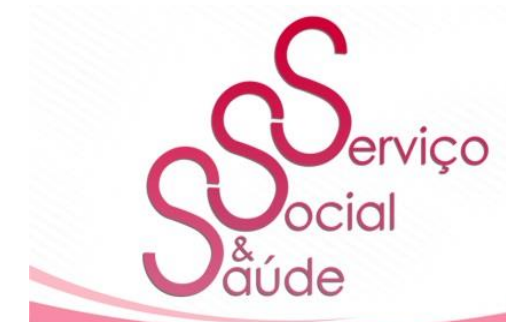

ISSN 2446-5992

(๑⿻)

10.20396/sss.v19i0.8665386

11

Portugal. O relato "a gente tenta adaptar essa escala para a nossa realidade, que a gente até tem uma dificuldade" (A3) se justifica, pois, a escala foi elaborada, de acordo com a realidade social e econômica da Espanha. Por esse motivo, foram realizadas adaptações na escala no estado de MS, porém ainda não foram suficientes para contemplar o contexto brasileiro.

A experiência do Serviço Social na Europa e no Brasil são distintas e, em nosso país, o Serviço Social desenvolve diferentes instrumentalidades participativas na relação com os usuários não tendo como tradição o uso de escalas de avaliação de realidades singulares. A partir do conteúdo das entrevistas com assistentes sociais da EGA pode-se afirmar que um dos critérios para inclusão no programa CCI é a presença de um cuidador familiar, como citado pelo participante A1 “essa família também precisa ser treinada, para poder cuidar desse paciente dentro da realidade social a qual se encontra" e pelo A3 "um dos principais critérios é o acompanhante, a família”, para acompanhar a pessoa internada e receberem treinamento, que serão necessários no domicílio.

Segundo Oliveira (2016) a família é entendida como a mais direta e imediata fonte de apoio social a pessoa que necessita de auxílio de terceiros. Portanto, a família é considerada o maior suporte àquele que necessita de apoio e cuidados. O processo de internação é um momento de tensionamentos para o usuário e família, pois além de provocar sentimentos de insegurança, medo e angústia afeta a dinâmica familiar, devido à ausência de um membro que está internado, e também daquele que irá acompanhá-lo. Entende-se que a presença familiar além de facilitar o elo entre a equipe e a pessoa internada, possibilita ao usuário maior segurança, apoio e afeto.

No entanto, a obrigatoriedade do acompanhamento traz diferentes impactos na dinâmica familiar, como tratado no estudo de Lima e Scandola (2018) sobre os impactos financeiros e de reordenamento familiar durante a internação na Unidade CCI, posto que a pessoa internada por vezes é o principal provedor da família, ocasionando impactos financeiros, em que os familiares necessitam assumir as responsabilidades; para permanecer 


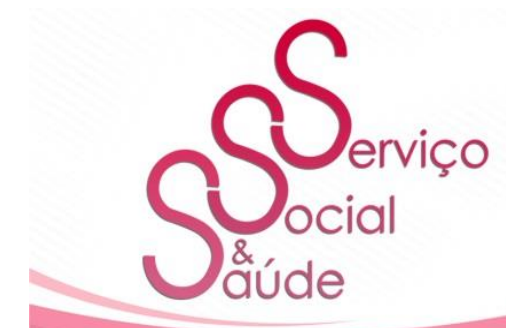

ISSN 2446-5992

(๑⿻)

10.20396/sss.v19i0.8665386

12

como cuidador um familiar teve que parar de trabalhar e/ou mudança de emprego; a internação resultou em aumento na carga de trabalho; benefícios sociais e previdenciários mantiveram a renda familiar.

Algumas famílias realizam a contratação de um cuidador formal para permanecerem como acompanhante no período de reabilitação do CCI, para contemplar o critério do Programa. Tal contratação de cuidador formal, por vezes será apenas no período de internação, o que contradiz o objetivo de capacitação para o domicílio. Além disso, essa condição é contrária ao previsto na PNHOSP em seu art. 10, em que após o acesso à atenção hospitalar a equipe de saúde será integralmente responsável pelo usuário a partir do momento de sua chegada (BRASIL, 2013).

Outra situação emergente com a obrigatoriedade do cuidador, é que na ausência desses, como nos casos de pessoas com vínculos familiares rompidos, que tinham uma vida independente antes do adoecimento, é que não podem participar do Programa, mesmo que contemple os demais critérios. Essa metodologia que "obriga" a família dispor de cuidador/familiar contradiz os princípios do SUS, seja na sua responsabilidade sobre as pessoas internadas ou na exclusão de direitos daqueles que não tem vínculos com os mesmos.

Ainda sobre os critérios usados, dois (2) entrevistados citaram o "protocolo" como base para sinalização dos usuários, como segue:

Bom, além do protocolo instituído pelo Hospital São Julião, a própria vivência mesmo, mas é mais o protocolo (E1).

A gente segue regulamentações e diretrizes impostas pelo Ministério da Saúde que classificam os pacientes de acordo com a realidade e com a capacidade de reabilitação (M2).

Os entrevistados E1 e M2 foram pontuais em tratar que utilizam o protocolo do Programa como base, sendo que o E1 citou o protocolo do HSJ e o M2 citou as regulamentações e diretrizes do Ministério da Saúde, sem especificar os critérios usados. 


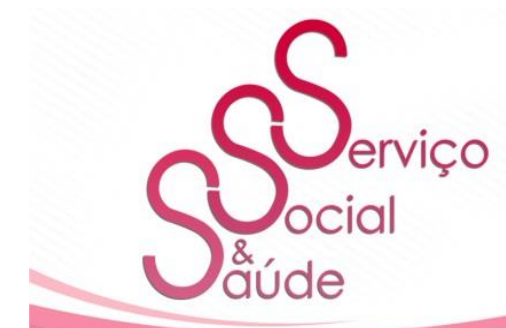

ISSN 2446-5992

(๑⿻)

10.20396/sss.v19i0.8665386

13

Segundo Werneck, Faria e Campos (2009, p. 31) “protocolos são as rotinas dos cuidados e das ações de gestão [...] que servem para orientar fluxos, condutas e procedimentos clínicos dos trabalhadores dos serviços de saúde”. No âmbito do Ministério da Saúde, os "Protocolos Clínicos e Diretrizes Terapêuticas" são documentos oficiais que estabelecem como devem ser realizados o diagnóstico, o tratamento e o acompanhamento dos pacientes (BRASIL, 2013 -2019).

A metodologia de atenção à saúde denominada CCI, ainda não é regulamentada como Programa em âmbito nacional, sendo assim, não existem "diretrizes e regulamentações" no SUS, a partir do Ministério da Saúde, específicas para essa modalidade de atenção. Mesmo sem uma tratativa legal específica para o CCI há que se considerar que a PNHOSP é suficiente para embasar uma gama de procedimentos a serem realizados nos hospitais.

A informação dada pelo M2 pode ter diferentes justificativas, sendo elas: desconhecimento do contexto histórico e legal do programa no Brasil; desconhecimento dos critérios de inclusão e exclusão do CCI, optando por citar o Ministério da Saúde; utilização de outras diretrizes e regulamentações no âmbito do SUS, dentre elas a regulamentação do NIR e/ou caracterizar o protocolo criado pelo HSJ como sendo do Ministério da Saúde.

No que se refere ao protocolo do serviço CCI instituído pelo HSJ, conforme citado pela E1, o documento se organiza em três (3) partes, sendo: 1 - operacionalização dos serviços, onde é detalhado como o programa se organiza (estrutura, período de atendimento; leitos disponibilizados, informações gerais); 2 - condições clínicas que caracterizam o paciente objeto do cuidado, sendo especificado o objetivo geral do programa e o perfil do paciente; 3 - aspectos inelegíveis à internação no CCI-HSJ, onde são apresentados os critérios de exclusão (HSJ, 2017), sendo que esse documento tem servido como base para as EGAs do estado.

\section{IDENTIFICAÇÃO DOS USUÁRIOS PARA A UNIDADE CCI}

Ao serem questionados se realizam busca ativa para identificação de pessoas com perfil para o CCI, os sete (7) participantes relataram que fazem busca ativa nos prontuários e

\begin{tabular}{|l|l|l|l|l|l|l} 
Serv. Soc. \& Saúde & Campinas, SP & v. 19 & $1-25$ & e020010 & 2020 & e-ISSN 2446-5992 \\
\hline
\end{tabular}




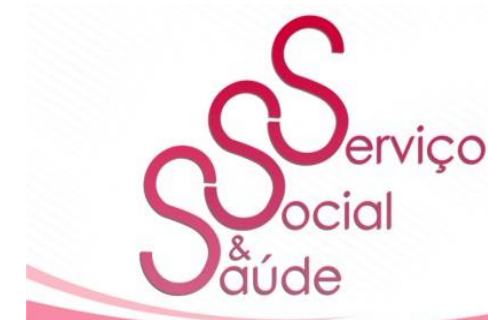

ISSN 2446-5992

(c) $10(0)$

10.20396/sss.v19i0.8665386

14

leitos, sendo que em dois (2) hospitais a EGA está inserida no NIR, ou seja, os profissionais que compõem a EGA também fazem parte do NIR. Dessa forma, os profissionais têm acesso a todas as movimentações de leitos interno e externo, o que facilita a identificação dos usuários elegíveis para o programa. O hospital que a EGA não é integrada ao NIR, realiza a busca ativa por meio do prontuário eletrônico, conforme relato da assistente social da referida instituição:

Nós fazemos busca ativa no censo hospitalar diariamente, e quando identificado, nós sinalizamos para equipe médica avaliar (A1).

Outra forma de identificação dos usuários para a unidade CCI citada pelos participantes é a partir da sinalização por parte da equipe de saúde, como se pode evidenciar nos discursos a seguir:

Por demanda do médico assistente que encaminha para o NIR (A1).

Quando ele entra no Pronto Socorro, já tem o médico que acompanha, e agora também tem outros médicos que já sabem e os residentes também colaboram nessa parte de sinalização (A2).

$\mathrm{Na}$ verdade, a maior parte das sinalizações acontece por parte dos residentes [...] hoje a gente depende das sinalizações praticamente dos residentes, tanto residentes médicos como multiprofissional, e os médicos da Neurologia Clínica sinalizam bastante os pacientes (M1).

A gente tem uma situação em que os médicos assistentes podem nos pedir via prescrição médica internamente [...]. Quem sinaliza normalmente é o enfermeiro que conhece o paciente. Então internamente a gente também faz essas reuniões de capacitação, e dissemina para o grupo. A gente tem nossa extensão com a equipe multiprofissional, então, eles também nos procuram, [...] nos sinalizam, mesmo se o médico assistente não sinalizar (E1).

Observa-se nos relatos que os diferentes trabalhadores em saúde, são fundamentais no processo de identificação e sinalização para o programa CCI. Haja vista que a EGA não atende diretamente as pessoas internados, sendo necessário o envolvimento da equipe em 


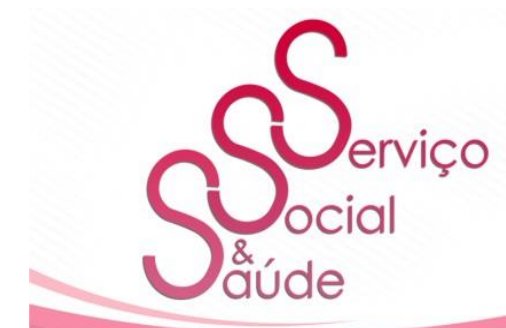

ISSN 2446-5992

(๑⿻)

10.20396/sss.v19i0.8665386

15

saúde para efetivação do programa CCI. Percebe-se no conteúdo do discurso que diferentes profissões podem ser sensibilizadas e capacitadas para o trabalho na rede CCI.

A sinalização dos usuários por parte da equipe médica e multiprofissional de acordo com os discursos se dá nas visitas beira-leito e via prontuário eletrônico, ferramentas essas, frequentes no âmbito hospitalar.

O participante E1 apresenta que são realizadas reuniões de capacitações para disseminar sobre o programa aos profissionais da instituição. Essa estratégia em saúde é norteada pela "Política Nacional de Educação Permanente" que se configuram como "uma proposta de aprendizagem no trabalho, onde o aprender e o ensinar se incorporam ao cotidiano das organizações", gerando reflexão sobre o processo de trabalho, mudanças institucionais e transformações nas práticas de serviço (BRASIL, 2013-2019).

Existem vários serviços e programas no âmbito do SUS voltados a promoção, reabilitação, habilitação e recuperação da saúde, que devem ser conhecidos por todos os trabalhadores em saúde, dentre eles o programa CCI. As ações de educação permanente propiciam aos profissionais, produção de conhecimento, atualização constante sobre processos, práticas e dinâmicas laborais, visando à efetivação dos princípios do SUS, dentre eles, promover a integralidade no acesso dos usuários do SUS aos serviços adequados para a sua necessidade de saúde.

Ainda na perspectiva da educação permanente os participantes A2 e M1 tratam sobre a participação dos residentes médicos e multiprofissionais no processo de sinalização. É importante salientar que os programas de residências são orientados pelos princípios e diretrizes do SUS, como uma modalidade de ensino em serviço de formação de trabalhadores para o SUS. Considera-se a residência em saúde como um espaço para o desenvolvimento das ações de educação permanente em saúde (SILVA et al., 2016).

É nos programas de residências que ocorrem diferentes práticas educativas, o que justifica a participação efetiva desses profissionais na identificação e sinalização de usuários com perfil para o Programa CCI, pois estão em constante aprendizado profissional. 


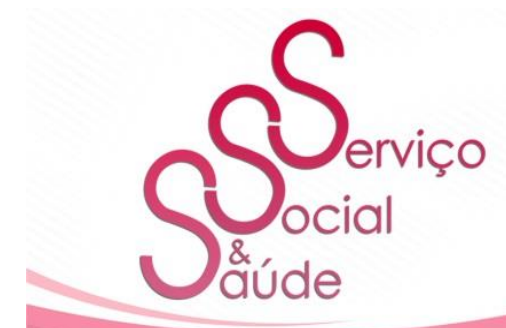

ISSN 2446-5992

(๑⿻)

10.20396/sss.v19i0.8665386

16

Podem-se afirmar a partir dos discursos que embora haja uma equipe mínima de profissionais da EGA, todos os profissionais em saúde dos hospitais que referenciam usuários para o Programa CCI são participantes e corresponsáveis no processo de sinalização para o programa CCI.

Além da busca ativa e sinalização pela equipe multiprofissional, um dos participantes relatou que atua na EGA e atende no pronto socorro da instituição, o que facilita a identificação de usuários, como segue:

Como eu sou plantonista do Pronto Socorro e sou daqui da EGA, a gente já vai sinalizando, desde o primeiro atendimento. Então, para mim é mais fácil triar, porque eu conheço os critérios de inclusão e exclusão. Quando eu atendo o paciente, eu já faço uma pré-triagem para reabilitação (M3).

De acordo com o Ministério da Saúde, pronto socorro é uma "unidade destinada à prestação de assistência a pacientes com ou sem risco de vida, cujos agravos necessitam de atendimento imediato" (BRASIL, 2008), com funcionamento 24 horas, na qual o usuário permanece em observação e/ou encaminhado para internação.

O pronto socorro como porta de entrada hospitalar, possibilita ao profissional que atende nessa unidade conhecer todos os usuários que deram entrada no hospital, sendo esse um facilitador para a identificação de pessoas para o Programa CCI.

Outro dado importante do relato da M3 é que quando o usuário é identificado no pronto socorro é possível realizar a preparação de alta para o CCI, desde a admissão hospitalar, que confere com o recomendado para o programa CCI de identificar de forma precoce as pessoas em situação de dependência, que pertençam ao grupo alvo, com a finalidade de planejar a alta desde o início da internação (DOLZ, 2013a).

\section{FLUXO DE SINALIZAÇÃO PARA A UNIDADE CCI}

Foi solicitado que os entrevistados descrevessem detalhadamente o fluxo de sinalização para a unidade CCI, sendo verificado que o primeiro procedimento realizado após a identificação dos usuários é a avaliação dos profissionais da EGA, como segue: 


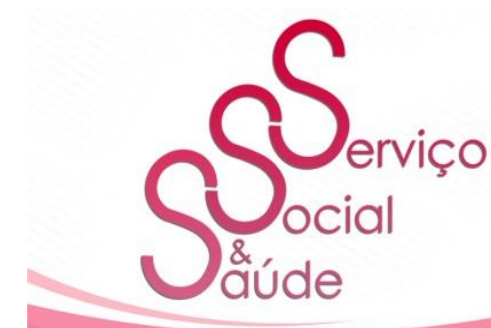

ISSN 2446-5992

(๑⿻)

10.20396/sSS.v19i0.8665386

17

A gente faz a avaliação médica e a avaliação do Serviço Social, já avaliação de Enfermagem está prejudicada por conta da ausência do profissional (M1).

Faço a parte médica, vejo como está, dou uma examinada, se tem arritmia ou não para ver se não é cardiopata, a gente aplica as escalas Barthel, Lawton, Minimentel e Braden, com o paciente e família, se ele está afásico, por exemplo, a gente usa muito a avaliação da família. Depois de aplicada as escalas, revisa o prontuário, como chegou, quais medicações foram feitas, se tem culturas positivas ou não (M2).

Faço visita ao leito, converso com a família, converso com o paciente, vê se eles aceitam, explicou como é o CCI, coloco como é a equipe, quais são os membros da equipe, como funciona, faço toda essa orientação (A1).

Nós do Serviço Social utilizamos a escala de Gijon (A2).

Após essa sinalização, a próxima avaliação é da equipe [EGA]. A médica que vai avaliar e dizer se esse paciente tem realmente perfil clínico para o CCI, posterior "entra" o Serviço Social para avaliar se esse paciente tem uma rede de apoio, se essa família vai ser corresponsável com essa internação (A3).

Observa-se a partir dos relatos que um dos procedimentos para avaliação das pessoas internadas para o CCI é a aplicação de diferentes instrumentos, as denominadas escalas de avaliação, sendo elas: Barthel, Lawton, Braden, Mini mental e Gijon. Tratando-se de um recurso em saúde, as escalas são construídas por meio de "referências teóricos e são validadas através de rigorosas análises estatísticas, que tem a capacidade de gerar, de modo coeso, dados sobre o provável estado de saúde de alguém ou de uma situação" (GARDONA; BARBOSA, 2018, p. 1921).

As escalas citadas são utilizadas para medir o desempenho das atividades de vida diária, risco de lesão por pressão, estado mental e situação sociofamiliar de idosos, respectivamente. Essas escalas foram incorporadas ao CCI a partir de Portugal e Espanha e passaram a ser utilizadas no Brasil sendo que sua maioria já são validadas, salvo a escala de Gijon utilizada pelo Serviço Social. 


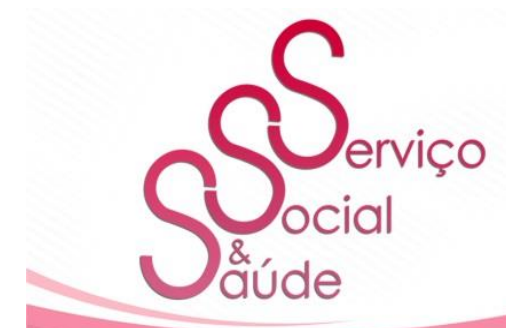

ISSN 2446-5992

(๑⿻)

10.20396/sss.v19i0.8665386

18

Os entrevistados M2 e A3 apresentaram alguns critérios que são usados na avaliação profissional "vejo como está, dou uma examinada, se tem arritmia ou não para ver se não é cardiopata", "quais medicações foram feitas, se tem culturas positivas ou não" e "se esse paciente tem uma rede de apoio". Esses critérios estão de acordo com o protocolo do HSJ, ou seja, são considerados inelegíveis para internação os usuários que apresentam infecção em atividade. Além disso, foi criado o "Protocolo para Prevenção e Precaução de Contra Infecções Hospitalares", com orientações para todas as unidades hospitalares envolvidas no CCI (HSJ, 2015-2016).

Outra situação são os usuários cuja restrição para o CCI é a ausência de cuidador e/ou rede de apoio, o que justifica o critério citado pelo A3. Quanto à arritmia para verificar se é cardiopata, os documentos de capacitação do programa e o próprio protocolo, não citam esse critério. No que se refere à ordem de avaliação, observa-se que não há uma regra específica, podendo ocorrer em conjunto, conforme relatado pelo M2 ou em momentos distintos como relatado pelo A3. Outro aspecto importante, citado pela participante A1 diz respeito ao esclarecimento sobre o programa ao usuário e família, para verificar o interesse em participar do CCI.

Um dos critérios de exclusão proposto no período de implantação do programa CCI é a "recusa por parte do paciente e/ou família, tutor legal e cuidador" (DOLZ, 2013b). Embora esse critério não esteja descrito no protocolo do HSJ, a "Carta dos Direitos dos Usuários da Saúde”, aprovada no Conselho Nacional de Saúde, assegura aos usuários do SUS, dentre outros aspectos, o direito ao "consentimento ou recusa de forma livre, voluntária e esclarecida, depois de adequada informação, a quaisquer procedimentos diagnósticos, preventivos ou terapêuticos" (BRASIL, 2007).

Partindo desse pressuposto, os usuários e familiares devem ser orientados quanto ao programa e ter autonomia de aceitar ou não a transferência para a unidade CCI. Após as 


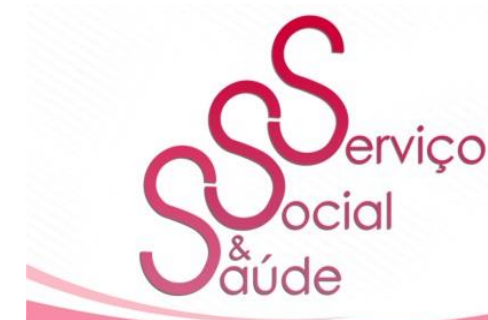

ISSN 2446-5992

(c) $10(0)$

10.20396/sss.v19i0.8665386

19

avaliações e aceitação do usuário, tendo critério para o Programa CCI é iniciado o processo de preenchimento e encaminhamento da pré-alta, conforme discursos dos participantes.

[A gente] encaminha para o São Julião através de e-mail, em até 24 horas, no máximo, eles $[\mathrm{HSJ}]$ já encaminham a resposta e, depois disso, o paciente vai (M1).

Normalmente a gente põe observação [na pré-alta], se está terminando antibiótico, se está programado algum ambulatório, nós ficamos a disposição deles [HSJ]. (M2).

É feita a pré-alta, é preenchida toda a documentação a ser enviada via e-mail, aí cada profissional envolvido no processo preenchia esse formulário [...] se a gente tinha alguma dúvida, os outros profissionais da unidade [...] HSJ falando que esse paciente estava apto, o hospital recebia uma senha por email através da regulação, esse paciente era encaminhado, reservava-se o transporte certinho (A2).

Existindo a vaga é preenchido a pré-alta pela equipe médica e Serviço Social, e encaminhamos para o São Julião. Então, nós usamos três ferramentas: via e-mail, contato telefônico e um grupo whatsapp [...] quando encaminhamos a pré-alta a equipe médica, encaminha todas as informações relacionadas a exames que o paciente fez aqui: exames de imagem, tomografia, ressonâncias, exames laboratoriais, evolução médica, medicação que está fazendo uso no momento. Na possibilidade de ser confirmada a vaga [...] vamos ao leito, orientamos essa família desse paciente que já está tudo certo, que a vaga está confirmada, e a importância da família estar aqui (A3).

A gente preenche toda a pré-alta cada um da equipe. Nesse momento ocupa o fisioterapeuta, a fonoaudióloga e o psicólogo da unidade, que tem aquela vivência com o paciente [...] a gente envia para avaliação. Tendo o aceite deles [HSJ], a gente reúne toda a [...] normalmente o CCI nos diz o melhor horário para admissão (E1).

Pode-se afirmar que o fluxo usado nos três hospitais é similar, sendo que as duas (2) assistentes sociais e a enfermeira relataram mais detalhadamente o processo. Em contrapartida, o participante M3 relatou:

Isso aí, quem vai saber é a enfermeira, [ela] vai precisar te responder mais detalhadamente. O que eu tenho conhecimento é que a gente faz a v. 19 1- 25 e020010 2020 e-ISSN 2446-5992 


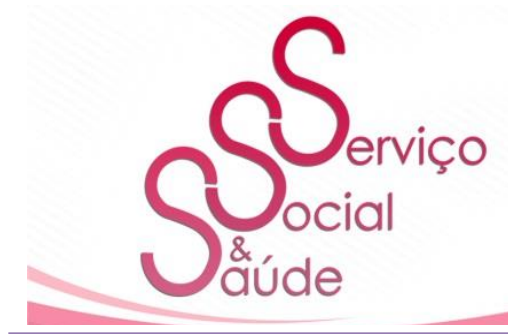

ISSN 2446-5992

(๑⿻)

10.20396/sss.v19i0.8665386

20

identificação desses pacientes, preenche um formulário que tem os requisitos e pré-requisitos, e é enviado para o CCI para eles avaliarem a possibilidade de transferência desse paciente, mas o fluxo detalhado a gente vai precisar ver com a enfermeira da regulação mesmo (M3).

Diante do discurso detalhado das participantes A2, A3 e E1, pode-se presumir que as assistentes sociais e a enfermeira participam mais ativamente do processo de encaminhamento do formulário de pré-alta para a EGA do HSJ, ou seja, das relações interinstitucionais.

É possível analisar que há um movimento dos profissionais em garantir a desospitalização de forma que beneficie o usuário com a continuidade do cuidado que atenda suas necessidades em saúde e ao hospital de alta complexidade, na medida em que promove a liberação do leito para pessoas com perfil clínico para internação. Carvalho (2014, p. 267) discute que a gestão de alta em Cuidados Continuados não é um processo fácil, pois demanda uma articulação em rede que requer "a elaboração de informações e relatórios escritos que validem as informações [...] Este processo é importante para não só validar as evidências, mas também para responsabilizar as instituições pelo processo de intervenção", o que justifica o fluxo adotados pelas EGAs dos hospitais na sinalização para o CCI.

Em relação ao processo de trabalho entre as equipes do hospital de Alta Complexidade e o do CCI, ocorrem etapas que podemos assim especificar: a) é realizado o preenchimento da pré-alta, onde os profissionais da EGA registram as informações levantadas na avaliação e, quando necessário são solicitadas a colaboração das outras especialidades que não compõe a EGA, mas que são importantes visando promover a segurança do usuário e o trabalho da equipe do hospital de destino; b) o formulário de pré-alta é encaminhado via e-mail para a EGA do HSJ.

Segundo Hadad e Jorge (2018, p. 206) a garantia da continuidade do cuidado "depende da transferência de informações para subsidiar decisões com relação ao tratamento". Por isso, a elaboração do relatório de pré-alta é realizada de forma sistemática, visando promover elementos necessários aos profissionais que irão receber o usuário no HSJ. São utilizados 


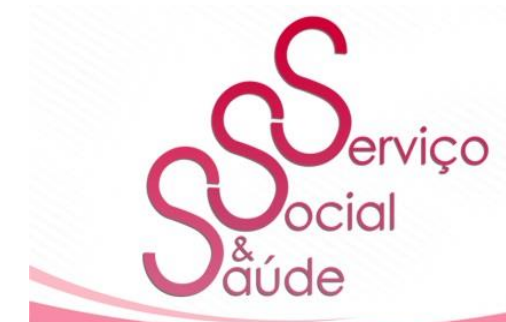

ISSN 2446-5992

(๑⿻)

10.20396/sSS.v19i0.8665386

21

também outros meios para comunicação entre a EGA demandante e a EGA executora, sendo eles: contato telefônico e aplicativo de mensagens (whatsapp); c) é agendado o transporte (responsabilidade do hospital que encaminha o usuário); d) a orientação familiar é realizada desde a sinalização, sendo que no momento de alta do hospital demandante há uma abordagem final pelo Serviço Social visando dirimir as dúvidas e informar quanto aos documentos e pertences necessários para serem levados para a unidade CCI, assim como, os contatos do hospital de destino.

\section{CONSIDERAÇÕES FINAIS}

A pesquisa realizada permite afirmar que as três (3) EGAs dos hospitais de Alta Complexidade de Campo Grande que foram capacitadas para encaminhar os usuários para a Unidade CCI continuam ativas, sendo compostas pela equipe mínima de profissionais, conforme período de implantação do programa no estado, com a ressalva da ausência do profissional de enfermagem em dois (2) hospitais.

É nesse contexto que foi evidenciado que a composição dos profissionais e o objetivo das EGAs, definição essa advinda do modelo português, são compatíveis ao definido para o Núcleo Interno de Regulação (NIR) do SUS, sendo que em dois (2) hospitais pesquisados os profissionais da EGA são os mesmos da equipe do NIR, o que facilita a identificação dos usuários com elegibilidade para diferentes programas, pós-internação hospitalar. Portanto, é perfeitamente cabível que o NIR exerça suas funções e não se crie instâncias sobrepostas como as EGAs e os NIR, ou seja, o Brasil já dispõe de um instrumento com regulamentação, não sendo necessário modelo externo.

Observou-se que embora haja as profissões de referência da EGA, outras especialidades em saúde participam do processo de identificação, avaliação e encaminhamento, fomentando uma visão multiprofissional sobre as necessidades em saúde dos usuários e encaminhamento a serviços com modelos de atendimento como é o CCI. Desse 


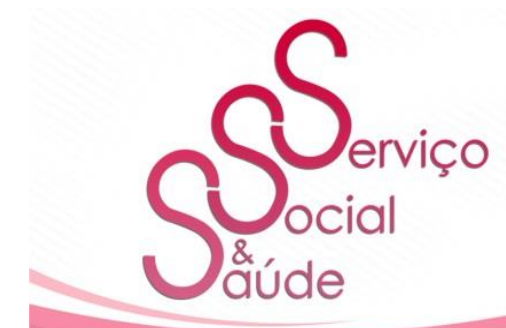

ISSN 2446-5992

(๑⿻)

10.20396/sss.v19i0.8665386

22

modo, deveria a regulamentação contar com a equipe mínima obrigatória e equipe sugerida como farmacêuticos, fisioterapeutas e nutricionistas.

No que se refere aos critérios utilizados para encaminhamento dos usuários ao CCI, os participantes citaram: a) perda da autonomia que, de acordo com os relatos é caracterizado pela perda da funcionalidade com possibilidades de reabilitação; b) a presença de rede de apoio sociofamiliar, pois os familiares participam efetivamente do processo de reabilitação e/ou de habilitação, visto que são realizadas capacitações na Unidade CCI aos familiares/cuidadores sobre diferentes temáticas inerentes à saúde; e, c) utilização de protocolos.

Quanto ao fluxo de sinalização foi verificado que ocorrem de forma equivalente entre os três hospitais, sendo realizada identificação, avaliação e encaminhamento pelos mesmos mecanismos. Vale destacar, que nos hospitais de Alta Complexidade é fundamental que ocorram capacitações de cuidadores e familiares sobre o cuidado específico e os direitos sociais, assim como, os direitos dos usuários do SUS. Verificou-se que alguns dos atuais critérios de encaminhamento podem ser conflituosos com os princípios do SUS, considerando os direitos conquistados e a realidade da população brasileira. Em situações de ausência de rede de apoio há que se considerar a importância das articulações intra e intersetoriais visando à garantia de direitos, especialmente a universalidade no SUS.

Outro aspecto significativo refere ao envolvimento da Política Nacional de Humanização e Política Nacional de Educação Permanente no contexto do Programa CCI no estado de MS, políticas essas, que não foram tratadas no período de capacitação para a implantação do Programa, mas estão intrínsecas ao SUS. Em virtude dos fatos mencionados, pode-se afirmar que o objetivo da EGA no Programa CCI é promover uma alta responsável, na perspectiva da mudança da centralidade do cuidado dentro da rede SUS.

A metodologia CCI é estritamente compatível com a PNHOSP e, portanto, resultado dos avanços realizados no SUS pelas pressões dos movimentos populares, a participação de usuários e trabalhadores nos Conselhos de Saúde e no exercício cotidiano do cuidado. O que 


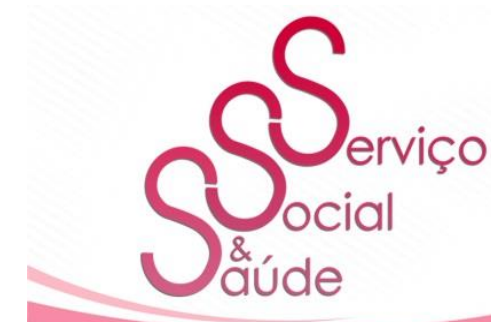

ISSN 2446-5992

(๑⿻)

10.20396/sss.v19i0.8665386

23

foi conquistado até 2013, ano de inauguração do Programa CCI no HSJ, precisa seguir e expandir-se. A defesa do CCI como resposta do SUS ao envelhecimento da população e ao cuidado interdisciplinar aos usuários que dele necessitam é uma possibilidade de avançar na humanização do setor hospitalar, do trabalho em rede e dever do SUS solidário, de qualidade e verdadeiramente público.

Recebido em dezembro de 2019 - Aprovado em julho de 2020

\section{REFERENCIAS}

ALVARENGA, A. F. O Projeto Piloto para Implantação dos Cuidados Continuados Integrados. In: DOBASHI, B. F. (Org.). Tecendo Redes na Saúde para Ampliar o Cuidado. Campo Grande - MS. 2014.

ARAÚJO, A. A.; BRITO, A. M.; NOVAES, M. Saúde e Autonomia: Novos conceitos são necessários? Revista Bioética. 16, n. 1, p. 117 - 124, 2008.

BRASIL. Cadastro Nacional de Estabelecimentos de Saúde. CNES. Brasília, DF. 2008. BRASIL. Constituição da República Federativa do Brasil. Brasília DF. 1988.

BRASIL. Lei no 8.080, de 19 de setembro de 1990. Dispõe sobre as condições para promoção, proteção e recuperação da saúde, a organização e o fundamento dos serviços correspondentes, e dá outras providências. Diário Oficial da República Federativa do Brasil, Brasília, DF, 20 set. 1990 .

BRASIL. Ministério da Saúde. Carta dos direitos dos usuários da saúde / Ministério da Saúde. 2. ed. Brasília: Ministério da Saúde, 2007. 9 p. (Série E. Legislação de Saúde). BRASIL. Ministério da Saúde. Política Nacional de Educação Permanente (PNEPS). Trabalho, educação e qualificação. Brasília, DF. 2013 - 2019.

BRASIL. Ministério da Saúde. Portaria nº 2.395, de 11 de outubro de 2011. Organiza o componente hospitalar da rede de atenção às urgências e emergências. Brasília, DF. 2011.

\begin{tabular}{l|l|l|l|l|l|l} 
Serv. Soc. \& Saúde & Campinas, SP & v. 19 & $1-25$ & e020010 & 2020 & e-ISSN 2446-5992 \\
\hline
\end{tabular}




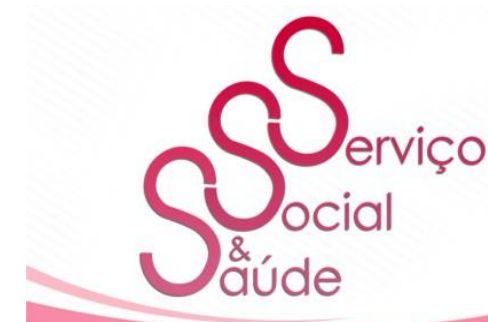

ISSN 2446-5992

(1) (10)

10.20396/sss.v19i0.8665386

BRASIL. Ministério da Saúde. Portaria n 2.528, de 19 de outubro de 2006. Aprova a

Política Nacional de Saúde da Pessoa Idosa. Brasília: Ministério da Saúde, 2006.

BRASIL. Ministério da Saúde. Portaria no 2.809 de 7 de dezembro de 2012. Organização dos

Cuidados Prolongados para retaguarda à rede de atenção as urgências e emergências.

BRASÍLIA. DF, 2012.

BRASIL. Ministério da Saúde. Portaria no 3390, de 30 de dezembro de 2013. Institui a

Política Nacional de Atenção Hospitalar (PNHOSP) no âmbito do Sistema Único de

Saúde (SUS); Brasília, DF, 2013.

BRASIL. Ministério da Saúde. Protocolo Clínico e Diretrizes Terapêuticas - PCDT.

Protocolos e Diretrizes. 2013 - 2019.

BRASIL. Ministério da Saúde. Resolução no 466, de 12 de dezembro de 2012. Aprovar as seguintes diretrizes e normas regulamentadoras de pesquisas envolvendo seres humanos. Brasília. DF. 2012.

BRASIL. Ministério da Saúde. Secretaria de Atenção à Saúde. Departamento de Atenção Hospitalar e de Urgência. Manual de implantação e implementação: Núcleo Interno de Regulação para Hospitais Gerais e Especializados [recurso eletrônico] / Ministério da Saúde, Secretaria de Atenção à Saúde, Departamento de Atenção Hospitalar e de Urgência. Brasília: Ministério da Saúde, 2017.

BRASIL. Política Nacional de Humanização. PNH. HumanizaSUS. Cartilha, 2013. CAMPOS, R. T. O; CAMPOS, G. W. S. Co-construção de autonomia: o sujeito em questão. Tratado de Saúde Coletiva; Editora Hucitec. 2006.

CARRARA, P.; DOLZ, M. Projeto: Implantação de Experiências de Cuidados

Continuados no Estado de Mato Grosso do Sul. 2013. [Não Publicado].

CARVALHO, M. I. L. B. Política de saúde e de cuidados continuados integrados em

Portugal. O planeamento da alta em Serviço Social. Rev. Katál., Florianópolis, v. 17, n. 2, p. 261-271, jul./dez. 2014.

CFESS. Parâmetros para Atuação de Assistentes Sociais na Política de Saúde. [Brasília]: 


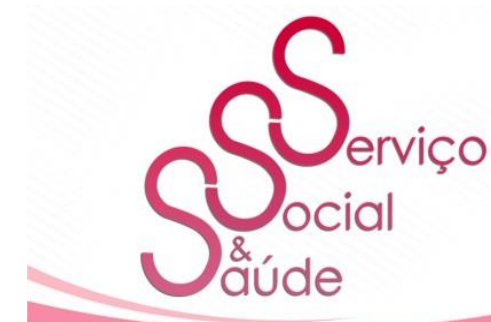

ISSN 2446-5992

(๑⿻)

10.20396/sss.v19i0.8665386

Conselho Federal de Serviço Social, 2010.

DOLZa, Montserrat. Preparação de Altas. Hospital Samaritano. CEALAG. GESAWORLD. SES. 2013 [Não Publicado].

DOLZb, Montserrat. Tipologias de Pacientes e Critérios de Admissão. Hospital

Samaritano. GESAWORDL. SES. 2013 [Não Publicado].

DOMINGUES, M. A. R. C; ORDONEZ, T. N; SILVA, T. B. L; NAGAI, P. A; BARROS, T.

C; TORRES, M. J. Revisão sistemática de instrumentos de avaliação de rede de suporte social para idosos. Revista Temática Kairós Gerontologia, v. 15, n. 6, p. 333-354, 2012.

GARDONA, R. G. B, BARBOSA, D. A. A importância da prática clínica apoiada em ferramentas de avaliação. Rev. Bras. Enferm. [Internet]. v. 71, n. 4, p. 1815, 2018.

HADAD, A. C. A. C; JORGE, A. O. Continuidade do cuidado em rede e os movimentos de redes vivas nas trajetórias do usuário-guia. Rio de Janeiro, v. 42, n. especial 4, p. 198-210, Dez, 2018.

HOSPITAL SÃO JULIÃO. HSJ. Protocolo para Prevenção e Precauções contra Infecções Hospitalares. Campo Grande. 2015-2016 [Não Publicado].

HOSPITAL SÃO JULIÃO. HSJ. Protocolo do Serviço de Cuidados Continuados

Integrados - CCI. Campo Grande, 2017 [Não Publicado].

LIMA, W. C. B; SCANDOLA, E. M. R. Os Impactos Financeiros na Vida dos Familiares

Durante a Internação na Unidade de Cuidados Continuados Integrados (UCCI). Rev. Saúde

Pública de Mato Grosso do Sul. v. 1, n. 1, p. 37-47, 2018.

MENDES, E. M. As Redes de Atenção à Saúde. Brasília: Organização Pan-Americana de Saúde, 2011.

MINAYO, M. C. S. (Org.). Pesquisa Social: teoria, método e criatividade. 28. ed. -

Petrópolis, RJ: Vozes, 2009.

OLIVEIRA, L. G. Contributos do Serviço Social no Acolhimento, Acompanhamento e Preparação da Alta de Doentes Internados em Unidades de Cuidados Continuados

Integrados. (Dissertação de Mestrado) - Universidade Fernando Pessoa. 2016. 


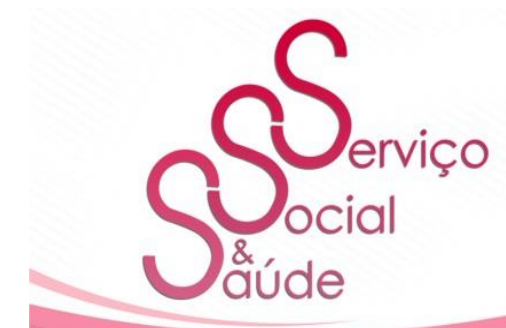

ISSN 2446-5992

(1) (10)

10.20396/sss.v19i0.8665386

PORTUGAL. Decreto-Lei no 101, de 06 de Junho de 2006. Rede Nacional de Cuidados Continuados Integrados. Portugal. 2006.

RIBEIRO, G. A.; SCANDOLA, E. M. R. Relação Entre Construção da Autonomia e da Independência Funcional do Sujeito de Ambiente Hospitalar a partir da Ação da Fisioterapia:

Uma Revisão de Literatura. In: CONGRESSO INTERNACIONAL DOS DIREITOS

HUMANOS, 2017, Campo Grande. Anais do XIV Congresso Internacional dos Direitos

Humanos. 2017.

SILVA, C. T; TERRA, M. G; KRUSE, M. H. L; CAMPONOGARA, S; XAVIER, M. da S.

Residência Multiprofissional como Espaço Intercessor para a Educação Permanente em

Saúde. Texto Contexto Enfermagem, v. 25, n. 1, 2016, p.1-9.

WERNECK, M. A. F; FARIA, H. P; CAMPOS, K. F. C. Protocolos de Cuidado à Saúde e de Organização do Serviço. Belo Horizonte: NESCON/UFMG, COOPMED, 2009. 